\title{
Is there a cost of virus resistance in marine cyanobacteria?
}

\author{
Jay T Lennon ${ }^{1}$, Sameed Ahmed M Khatana ${ }^{1}$, Marcia F Marston ${ }^{2}$ and Jennifer BH Martiny ${ }^{1,3}$ \\ ${ }^{1}$ Department of Ecology and Evolutionary Biology, Brown University, Providence, RI, USA and ${ }^{2}$ Department \\ of Biology and Marine Biology, Roger Williams University, Bristol, RI, USA
}

\begin{abstract}
Owing to their abundance and diversity, it is generally perceived that viruses are important for structuring microbial communities and regulating biogeochemical cycles. The ecological impact of viruses on microbial food webs, however, may be influenced by evolutionary processes, including the ability of bacteria to evolve resistance to viruses and the theoretical prediction that this resistance should be accompanied by a fitness cost. We conducted experiments using phylogenetically distinct strains of marine Synechococcus (Cyanobacteria) to test for a cost of resistance (COR) to viral isolates collected from Mount Hope Bay, Rhode Island. In addition, we examined whether fitness costs (1) increased proportionally with 'total resistance', the number of viruses for which a strain had evolved resistance, or (2) were determined more by 'compositional resistance', the identity of the viruses to which it evolved resistance. A COR was only found in half of our experiments, which may be attributed to compensatory mutations or the inability to detect a small COR. When detected, the COR resulted in a $\sim 20 \%$ reduction in relative fitness compared to ancestral strains. The COR was unaffected by total resistance, suggesting a pleiotropic fitness response. Under competitive conditions, however, the COR was dependent on compositional resistance, suggesting that fitness costs were associated with the identity of a few particular viruses. Our study provides the first evidence for a COR in marine bacteria, and suggests that Synechococcus production may be influenced by the composition of co-occurring viruses.
\end{abstract} The ISME Journal (2007) 1, 300-312; doi:10.1038/ismej.2007.37; published online 28 June 2007

Subject Category: microbial population and community ecology

Keywords: bacteriophage; evolution; host-parasite interactions; cyanophage; Synechococcus

\section{Introduction}

Viruses are abundant in marine environments, often exceeding the densities of their bacterial host populations (Proctor and Fuhrman, 1990; Weinbauer and Rassoulzadegan, 2004). Since viruses are obligately parasitic, it is assumed that they are important agents of mortality for microorganisms. Indeed, virus-induced mortality of bacteria can equal the losses imposed by protist and zooplankton grazing (Fuhrman and Noble, 1995). Such findings have led to the prevailing view that viruses are important for structuring microbial communities and regulating biogeochemical cycles (Fuhrman, 1999; Wilhelm and Suttle, 1999; Weinbauer, 2004;

Correspondence: Current address. JT Lennon, WK Kellogg Biological Station and Department of Microbiology and Molecular Genetics, Michigan State University, 3700 East Gull Lake Drive, Hickory Corners, MI 49060, USA.

E-mail: lennonja@msu.edu

${ }^{3}$ Current address: Department of Ecology and Evolutionary Biology, University of California at Irvine, Irvine, CA 92697, USA. Received 9 March 2007; revised 23 April 2007; accepted 23 April 2007; published online 28 June 2007
Bouvier and del Giorgio, 2007). In many studies, however, virus-induced mortality can be low (Fuhrman, 1999), possibly due to bacterial virus resistance.

The evolution of virus resistance has been welldocumented for clinically important strains of Escherichia, Salmonella and Pseudomonas (Chao et al., 1977; Lenski and Levin, 1985; Buckling and Rainey, 2002; Harcombe and Bull, 2005). In these systems, populations of virus-resistant bacteria rapidly evolve, invade the microbial community, and persist through time (Bohannan and Lenski, 2000). In contrast, few studies have documented the ease by which marine bacteria evolve virus resistance or its extent in marine systems. While some reports infer that resistance to marine viruses is rare (Fuhrman, 1999), others suggest that it may be widespread (Waterbury and Valois, 1993; Middelboe et al., 2001; Jiang et al., 2003; Sullivan et al., 2003).

The potential prevalence of virus resistance sets up a 'paradox' (Weinbauer, 2004): if virus resistance readily evolves, what prevents resistant bacteria from outcompeting sensitive bacteria and driving viruses extinct? Theory suggests that the coexistence 
of sensitive and resistant bacteria may be due to a trade-off between competitive ability and reduced mortality (Lenski, 1988b). Experimental evidence indicates that virus-resistant bacteria are usually less efficient competitors for growth-limiting resources than sensitive bacteria in the absence of viruses (Buckling and Rainey, 2002); in other words, there is typically a fitness cost associated with the evolution of virus resistance (Bohannan and Lenski, 2000). For lytic viruses, resistance is often conferred through the loss or configuration change of a receptor molecule on the bacterial cell surface, which interferes with attachment of a virus to its host (Inoue et al., 1995; Bohannan and Lenski, 2000). Such mutations often translate into a fitness cost via reduced rates of resource uptake (Bohannan et al., 1999). The magnitude of this cost of resistance (COR) reflects the host's genotype and its environment (Bohannan et al., 2002). For example, different mutations can impose varied costs (for example, T7 and T4 phage), but the COR can also be influenced by the resource environment (Bohannan et al., 1999).

The laboratory systems described above provide an excellent framework for exploring the COR in strains of environmental bacteria. Very little has been carried out, however, to explore the COR in marine bacteria, which presumably experience exposure to diverse viral assemblages. Recently, there have been major advances in our understanding of the ecology and evolution of environmental viruses through the study of marine cyanobacteria, especially Synechococcus (Waterbury and Valois, 1993; Suttle and Chan, 1994; Mann, 2003; Marston and Sallee, 2003; Paul and Sullivan, 2005). In addition to being a fairly well-characterized host-parasite system, understanding viral influence on Synechococcus populations is important due to its broad geographic distribution and functional role in marine ecosystems (Bertilsson et al., 2003).

To better understand how viruses affect marine bacteria, we addressed two main questions. First, does Synechococcus incur a fitness cost when it evolves resistance to a virus? Second, what factors influence the magnitude of a COR in Synechococcus? To address these questions, we measured the growth rates of multiple Synechococcus strains that had been selected for resistance to a suite of marine viruses (Stoddard et al., unpublished data). We then determined whether Synechococcus growth rates could be explained by breadth-of-resistance (BOR) profiles, which describe a host's sensitivity or resistance to a suite of genetically different viruses. We hypothesized that not only would virus resistance in Synechococcus incur a fitness cost, but that Synechococcus growth rates would decrease with each additional virus to which it evolved resistance. In other words, we predicted there would be a negative correlation between bacterial fitness and 'total resistance' (that is, the number of viruses in a BOR profile to which a strain is resistant). In addition, Synechococcus fitness may be influenced by the identity of the viruses to which it evolved resistance. If true, we expected that there would be a relationship between a Synechococcus strain's 'compositional resistance' (as defined by the multivariate characterization of Synechococcus BOR profiles) and its fitness.

\section{Methods}

Ancestral bacterial strains

Bacterial strains were derived from four ancestral strains of marine Synechococcus that were obtained from the Woods Hole Collection of Cyanobacteria (Woods Hole Oceanographic Institution, Woods Hole, MA, USA). Strains WH7803 and WH8012 were originally isolated from the Sargasso Sea, while WH8018 and WH8101 were isolated from coastal waters adjacent to Woods Hole, MA, USA (Waterbury et al., 1986). These Synechococcus strains vary in their pigment composition and can be distinguished based on 16S-23S rRNA internal transcribed spacer sequences (Rocap et al., 2002). WH8012 belongs to Synechococcus clade II and WH8018 belongs to Synechococcus clade VI, while WH7803 and WH8101 belong to distinct subclades within Synechococcus clade V. We used SN medium (seawater-based) for isolating Synechococcus strains and AN medium (artificial seawater) for subsequent culturing and experiments (Waterbury and Willey, 1988). These ancestral Synechococcus strains were maintained at room temperature on a 14:10 lightdark cycle at $10 \mu \mathrm{M} \mathrm{m}^{-2} \mathrm{~s}^{-1}$.

\section{Viral isolates}

A total of 32 marine viruses were isolated on one of the four ancestral Synechococcus strains. Detailed methods on the isolation and characterization of these viruses can be found in Marston and Sallee (2003). Briefly, seawater was collected from Mount Hope Bay, RI near Roger Williams University. Filtered seawater $(100 \mu \mathrm{l})$, dense Synechococcus culture $\left(100 \mu \mathrm{l}\right.$ of $\left.\sim 10^{8} \mathrm{cell} \mathrm{ml}^{-1}\right)$ and sterile SN media $(1000 \mu \mathrm{l})$ were combined in replicate wells of a microtiter plate. The plates were incubated under constant illumination for up to 3 week, while monitoring for lysis, which was noted by a clearing of the pigmented culture relative to control wells. Viruses were isolated from the lysates via plaque purification (Marston and Sallee, 2003). Resulting plaques were sterilely picked and reinoculated into a fresh culture of the original Synechococcus strain. The lysate produced from this last step was filtered $(0.45 \mu \mathrm{m})$ and used in batch culture experiments designed for the selection of virus-resistant Synechococcus. PCR amplification of the gene encoding for the capsid assembly protein (g20) indicated that all of the viruses in this study belong to the Myoviridae (Marston and Sallee, 2003). Genetic 
and phenotypic similarity analyses indicated that the viral isolates were distinct from one another (Stoddard et al., unpublished data). The viruses were named 'S-RIM' (Synechococcus-Rhode Island Myovirus) followed by the isolate number (for example, S-RIM36).

\section{Selection for virus-resistant bacteria}

We selected for virus-resistant bacteria by exposing ancestral Synechococcus strains to the isolated viral isolate (Stoddard et al., unpublished data). We combined $100 \mu \mathrm{l}$ of a dense Synechococcus culture ( $\sim 10^{8}$ cell $\mathrm{ml}^{-1}$ ), $10-40 \mu \mathrm{l}$ of plaque-purified virus and $1500 \mu \mathrm{l} \mathrm{SN}$ media in 24-well microtiter plates. We incubated the microtiter plates and examined the wells for cell lysis. By eye, we monitored the wells for regrowth of Synechococcus, which occurred in 4-10 week. From wells that regrew, single colonies were isolated via dilution culturing in SN soft agar (Brahamsha, 1996) and transferred to $50 \mathrm{ml}$ Erlenmeyer flasks containing SN media. We confirmed that the selected bacterial strains were virus resistant by challenging them against the original viral isolate in replicate microtiter wells. Further, in three instances, we sequentially selected for virus resistance using multiple viral isolates. In other words, we challenged a previously selected virusresistant Synechococcus strain with a novel virus, monitored for cell lysis and subsequent regrowth, and then isolated the new virus-resistant strain of Synechococcus.

In total, we isolated 22 virus-resistant strains of Synechococcus: seven from WH7803, four from WH8101, three from WH8012 and eight from WH8018. We named the resistant strains by adding ' $\mathrm{R}$ ' (for 'resistant') and the identifier of the S-RIM viruses (that is, 1-36) to the end of the ancestral strain. For example, WH8018 that became resistant to S-RIM8 was named 'WH8018R8'. In the case of 'WH8018R3R2R6', ancestral WH8018 was first selected for resistance to virus S-RIM3, then to S-RIM2, and finally to S-RIM6. Prophage was not induced with Mitomycin C (McDaniel and Paul, 2005) for any of the ancestral or virus-resistant Synechococcus strains (Stoddard et al., unpublished data).

\section{Breadth of resistance}

We characterized the phenotype of each Synechococcus strain by challenging it against each of the 32 viral isolates using the microtiter-plate approach described above. If a virus caused a Synechococcus strain to lyse within a 3-week period, then that bacterial strain was considered 'sensitive'. In contrast, if a Synechococcus strain did not lyse after being challenged with a virus, then the bacterial strain was considered 'resistant' to that particular virus. Thus, a BOR profile refers to the sensitivityresistance pattern of a Synechococcus strain to the 32 viral isolates (Stoddard et al., unpublished data).
Collectively, the pairwise crosses between all bacteria and all viruses resulted in a binary (sensitive $=0, \quad$ resistant $=1$ ) sensitivity-resistance matrix.

We characterized the BOR profile for each of the 25 Synechococcus strains in two ways. First, 'total resistance' refers to the summed number of viruses to which a Synechococcus strain was resistant (out of the 32 viral isolates). Second, 'compositional resistance' refers to the multivariate similarity of BOR profiles among different strains of bacteria. To quantify compositional resistance, we performed a Principal Coordinates Analysis (PCoA) on a similarity matrix generated from the sensitivity-resistance matrix. The similarity matrix used in the PCoA was a slight modification of the mean character difference (Legendre and Legendre, 1998):

$$
s_{i k}=1-\left[\frac{1}{p} \sum_{j=1}^{p}\left|y_{i j}-y_{k j}\right|\right]
$$

where $s_{i k}$ is the similarity of BOR profiles for Synechococcus strains $i$ and $k, j$ is the first of $p$ total viruses, and $y$ refers to the phenotypic observations for a given viral isolate. PCoA was performed in Matlab (version 6.3).

For the WH8018 strains, we constructed BOR profiles on two occasions; once when resistant strains were first isolated and again $\sim 12$ months later. The two sets of BOR profiles allowed us to evaluate the stability of the bacterial phenotypes over time. In particular, we were interested in determining whether a resistant phenotype reverted to a sensitive phenotype in the absence of virus.

\section{COR: growth curves}

We used two approaches to test whether Synechococcus experienced a cost associated with the evolution of virus resistance. The first approach compared the maximum growth rates $\left(\mu_{\max }\right)$ among 25 Synechococcus strains in growth curve experiments (Kassen and Rainey, 2004). We inoculated $1 \mathrm{ml}$ of a log-phase culture into an Erlenmeyer flask containing $20 \mathrm{ml}$ of sterile AN media. Each strain was grown in triplicate for a total of 75 experimental units. We incubated the culture flasks in a Percival growth chamber at $25^{\circ} \mathrm{C}$ on a 14:10 light-dark cycle at $10 \mu \mathrm{E} \mathrm{m}^{-2} \mathrm{~s}^{-1}$. As a surrogate for cell density, we monitored fluorescence (ex: $515 \mathrm{~nm}$, em: $570 \mathrm{~nm}$ for WH7803, WH8012 and WH8018 strains; ex: $515 \mathrm{~nm}$, em: $660 \mathrm{~nm}$ for WH8101) on $150 \mu \mathrm{l}$ subsamples from each culture every $24 \mathrm{~h}$ for 18 days using a Molecular Devices SpectraMax M5 plate reader.

A variety of models have been developed for estimating growth parameters from batch culture experiments (Baty and Delignette-Muller, 2004). We elected to use the modified Gompertz equation, because it is relatively simple, easy to interpret and has been shown to be effective in fitting empirical 
growth curve data (for example, Zwietering et al., 1990):

$$
y=A \exp \left\{-\exp \left[\frac{\mu_{\max } e}{A}(\lambda-t)+1\right]\right\}
$$

where $y$ is cell density (or in this case fluorescence), $A$ is the carrying capacity, $\mu_{\max }$ is maximum growth rate, and $\lambda$ is the lag time, which defines the duration of time between inoculation and the initiation of exponential growth (Zwietering et al., 1990). We fit $\log _{10}$ transformed fluorescence as a function of time using nonlinear regression (PROC NLIN, SAS version 8.01) with non-derivative parameter estimation (DUD).

We tested multiple hypotheses regarding the growth rates of the Synechococcus strains. First, we used one-way analysis of variance (ANOVA) to test whether there were differences in $\mu_{\max }$ among the four ancestral Synechococcus strains. Second, we tested the null hypothesis that all Synechococcus strains (ancestral and resistant) had similar $\mu_{\max }$ using one-way ANOVA with Tukey's post hoc comparisons of means (PROC GLM, SAS version 8.01). Third, we used one-way ANOVA with linear contrasts to test the $a$ priori hypothesis that resistant strains of Synechococcus would have lower $\mu_{\max }$ than the ancestral Synechococcus strains. Linear contrasts were used because they allow for flexibility in hypothesis testing and tend to be statistically more powerful than post hoc comparisons (Gotelli and Ellison, 2004). Fourth, we used linear regression (PROC GLM, SAS version 8.01) to test whether $\mu_{\max }$ decreased with the total resistance of a strain's BOR profile. Lastly, we tested whether $\mu_{\max }$ was affected by the compositional resistance of a strain's BOR profile using information generated from the PCoA. Specifically, we used the eigenvectors corresponding to the first three principal coordinate axes as independent predictors of Synechococcus $\mu_{\max }$; if there was a non-zero slope between the eigenvectors and bacterial growth rate, then we concluded that compositional resistance affected Synechococcus fitness. In addition, we calculated correlation coefficients for the principal coordinate scores generated from each PCoA axis against the resistance-sensitivity profile of each viral isolate. In this case, a strong positive or negative correlation suggests that a viral isolate was influential in a PCoA.

\section{COR: competition assays}

A second strategy for measuring fitness trade-offs is to directly compete sensitive and resistant strains of bacteria. Although logistically more difficult, this approach is often preferred because it integrates abiotic and biotic factors that influence a population's ability to persist through time (Kassen and Rainey, 2004). One obstacle with the competition approach is the challenge of distinguishing between morphologically similar strains in co-culture. We addressed this issue by conducting assays where ancestral and virus-resistant Synechococcus were competed separately against a reference strain (de Roode et al., 2005).

Although ecologically similar, certain clades of Synechococcus can be distinguished from one another based on their phycobiliprotein composition (Stomp et al., 2004). For example, all strains of WH7803, WH8018 and WH8012 contain the accessory pigment phycoerythrin. In contrast, WH8101 lacks phycoerythrin, but instead contains phycocyanin. Therefore, we assessed the competitive ability of our target strains (ancestral and virusresistant phycoerythrin-containing strains) by competing them against a reference strain (ancestral phycocyanin-containing WH8101).

We initiated competition assays by inoculating replicate Erlenmeyer flasks containing $25 \mathrm{ml}$ of sterile AN media with equal densities of the target and reference strains. Initial densities of each population were $\sim 10^{6} \mathrm{cell} \mathrm{ml}^{-1}$. Cultures were incubated in a Percival growth chamber at $25^{\circ} \mathrm{C}$ on a 14:10 light-dark cycle at $10 \mu \mathrm{E} \mathrm{m}^{-2} \mathrm{~s}^{-1}$ for 7-10 days. Samples from each flask were taken at the beginning $\left(t_{0}\right)$ and end $\left(t_{f}\right)$ of the experiment by filtering samples onto $0.2 \mu \mathrm{m}$ black polycarbonate filters (Osmonics). Filter images were captured using a Zeiss Axioplan II microscope and Axiocam MRm camera. From these images, we estimated total Synechococcus densities (phycoerythrin+ phycocyanin-containing strains) using a CY5 filter set (ex: $640 \mathrm{~nm}$; em: $680 \mathrm{~nm}$ ). We quantified phycoerythrin-containing Synechococcus (that is, target population) using a fluorescein isothiocyanate (FITC) filter set (ex: $480 \mathrm{~nm}$; em: $535 \mathrm{~nm}$ ). We then estimated cell densities of the reference strain as the difference between CY5 and FITC counts. We calculated Synechococcus growth rates as $\left[\ln \left(\mathrm{N} t_{f}\right) /\right.$ $\left.\ln \left(\mathrm{N} t_{0}\right)\right] / t$, where $\mathrm{N}$ is Synechococcus density $\left(\right.$ cell $\mathrm{ml}^{-1}$ ). Relative fitness was expressed as the ratio of resistant to ancestral growth rates.

We performed two competition assays to test different COR hypotheses. The first was designed to determine whether the COR was dependent on the concentration of a growth-limiting resource. Before initiating the assay, we grew up batch cultures of an ancestral population (WH7803), a virus-resistant population (WH7803R8) and the reference population (WH8101) in either low $(0.9 \mu \mathrm{M})$ or high $(90 \mu \mathrm{M})$ phosphate concentrations through two serial transfers. Nitrogen, supplied as $\mathrm{KNO}_{3}$, was held at a constant N:P ratio of 100 . We analyzed the growth rate data using two-way ANOVA (factor $1=$ phosphorus concentration, factor $2=$ strain phenotype, PROC GLM, SAS version 8.01). Each treatment combination was replicated in triplicate.

The second assay was designed to test whether the COR of a strain was related to its BOR profile. We used WH8018 for this assay because, compared to the other Synechococcus groups, it contained the 
largest number of strains in our collection and it exhibited the most phenotypic variation in its sensitivity-resistance matrix. Each competition trial (one of the nine WH8018 strains vs the reference strain) was replicated five times. We used the same set of hypotheses and statistical analyses for the competition data that we used for the growth curve experiments.

\section{COR: assessing detection limits}

We performed power analyses (JMP software, version 5.1) for the growth curves experiments and competitive assays to evaluate our ability to detect relative fitness costs ranging from 2.5 to $50 \%$. This range in COR represents the 'effect size' in the power analyses. We used within-treatment standard deviations as an estimate of our measurement error. The probability of committing a Type I error (rejecting the null hypothesis when it is true) is expressed as $\alpha$, while the probability of committing a Type II error (accepting the null hypothesis when it is false) is expressed as $\beta$. We assumed a typical $\alpha$ value of 0.05 and then estimated power $(1-\beta)$ for a range of experimental replication $(n=3,5$ and 10).

\section{Results}

Breadth of resistance

The ancestral and virus-resistant Synechococcus strains investigated in this study displayed substantial phenotypic diversity in the form of their BOR profiles. A detailed treatment of the BOR data can be found in Stoddard et al. (unpublished data). Briefly, total resistance, which we define as the number of viral isolates to which a bacterial strain is resistant, ranged from 10 to 15 for WH8101 strains, 13 to 21 for WH8012 strains, 2 to 13 for WH7803 strains and 3 to 30 for WH8018 strains (Table 1 and Figure 1). The compositional profiles of virus resistance varied among strains as well. For example, despite having the same total resistance, the BOR profiles for WH8101R22 and WH8101R3 were only 75\% similar (Table 1). The bacterial strains were distinct from one another based on variation in their BOR profiles, as indicated by the amount of variance explained in the PCoA (Table 2 and below).

BOR profiles for the WH8018 strains were relatively stable over time; $95 \%$ of the potential 288 interactions (nine bacterial strains $\times 32$ viral isolates) remained fixed over a 12-month period. In addition, there was no apparent directionality for the 15 observed changes; $53 \%(n=8)$ switched from resistant to sensitive (reversion), and $47 \% \quad(n=7)$ switched from sensitive to resistant. Interestingly, six of the eight reversions were associated with only two of the 32 viruses (S-RIM28 and S-RIM32, Table 1).

\section{COR: growth curves}

Before analyzing growth curve data for the COR hypotheses, we noted that the maximum growth rates $\left(\mu_{\max }\right)$ of ancestral Synechococcus strains were not equal among the four phylogenetic groups $\left(P<0.0001\right.$, Figure 1b). In particular, the $\mu_{\max }$ of WH8101 $(0.59 \pm 0.023)$ was significantly higher than that of the three other ancestral strains $(0.33 \pm 0.061)$. Therefore, subsequent tests of the COR hypotheses were performed for each group separately.

Synechococcus $\mu_{\max }$ was only affected by virus resistance in the WH8101 group, which happened to have the highest maximum growth rates. A one-way ANOVA followed by Tukey's post hoc comparison of means indicated that the $\mu_{\max }$ of ancestral WH8101 was significantly greater than that of WH8101R27 $\left(P_{\text {null }}=0.014\right.$, Figure 1). Furthermore, linear contrasts testing for differences in $\mu_{\max }$ between the ancestral and all virus-resistant strains revealed that there was a significant COR $\left(P_{\text {contrast }}=0.0019\right.$, Figure 2) equating to an average $18 \%$ reduction in relative fitness.

We did not detect a COR in the remaining three groups of Synechococcus based on our growth curve data (Figure 1). A one-way ANOVA revealed that $\mu_{\max }$ were not equal among all WH8018 strains $\left(P_{\text {null }}=0.029\right.$, Figure 1$)$, but post hoc comparisons of means indicated that this was due to one virusresistant strain (WH8018R34) having a higher $\mu_{\max }$ than another virus-resistant strain (WH8018R22). A subsequent linear contrast within this phylogenetic group revealed that the ancestral and virus-resistant WH8018 strains had similar $\mu_{\max }$ values $\left(P_{\text {contrast }}=0.352\right.$, Figure 1$)$. Likewise, there were no significant differences in $\mu_{\max }$ for the WH7803 or WH8012 strains $\left(P_{\text {null }}\right.$ and $P_{\text {contrast }} \geqslant 0.118$, Figure 1$)$.

In general, BOR characterizations were poor predictors of $\mu_{\max }$. Contrary, to our prediction, $\mu_{\max }$ did not decline with increasing total resistance for any of the strains belonging to the four Synechococcus groups (linear regression, Table $1, P \geqslant 0.453$ ). Similarly, we found no relationship between $\mu_{\max }$ and compositional resistance (Table 1). Although a large fraction of the BOR variation was explained via the multivariate (PCoA) analyses (Table 1), Synechococcus $\mu_{\max }$ could not be explained by eigenvectors corresponding to its first three principal coordinates axes (Table $1, P \geqslant 0.146$ ). This result means there was no relationship between a strain's fitness (as defined by $\mu_{\max }$ ) and the combination of viruses to which it was resistant (that is, compositional resistance).

\section{COR: competition assays}

Results from the first competition assay indicated that a virus-resistant strain of Synechococcus (WH7803R8) was competitively inferior compared to ancestral Synechococcus (WH7803) $(P=0.036$, resistance main effect, two-way ANOVA, Figure 2). In addition, Synechococcus growth rates were 
Table 1 Sensitivity-resistance matrix depicting the phenotypes of the nine Synechococcus strains from the WH8018 clade

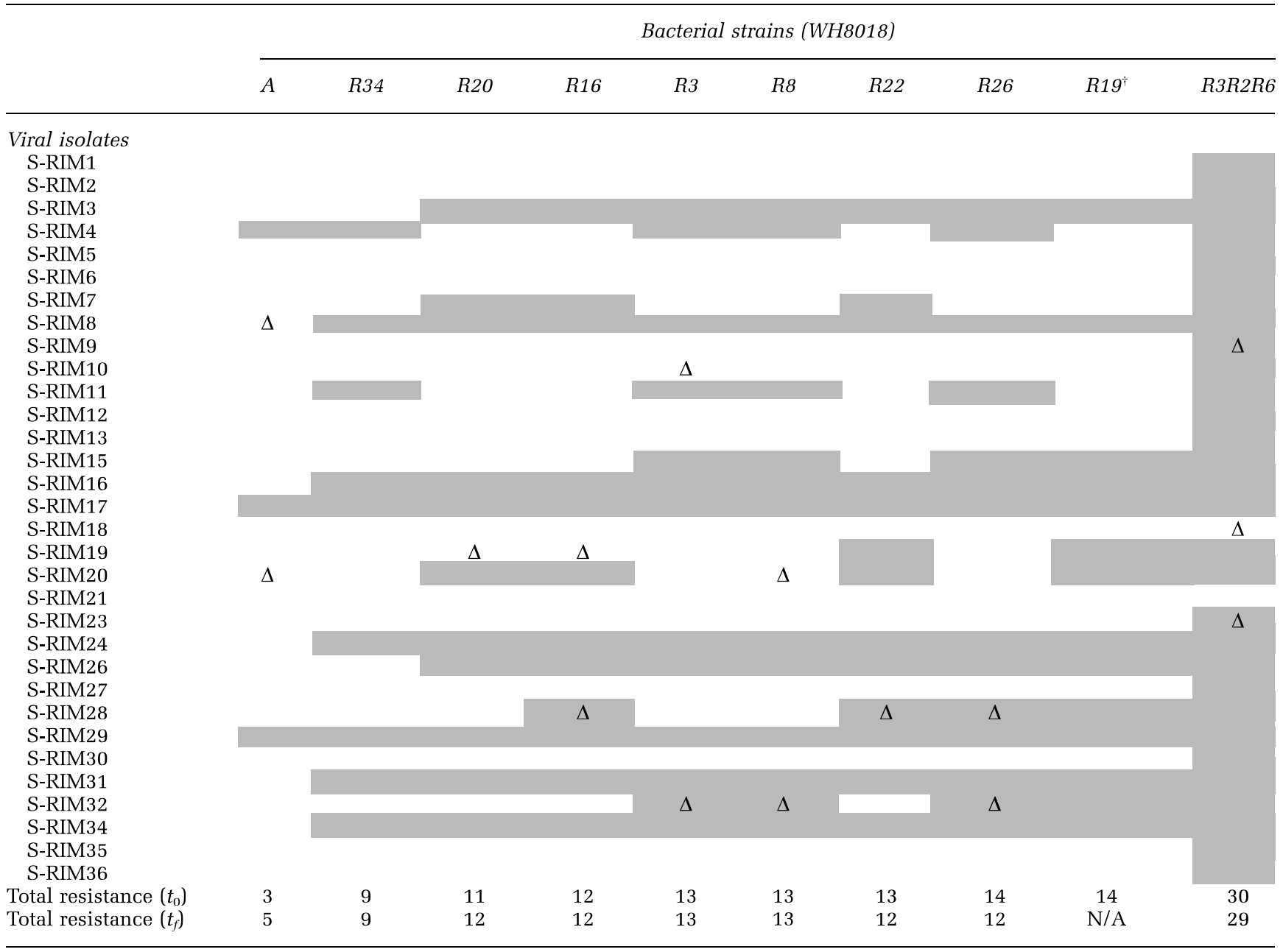

For the bacterial strains, 'A' refers to the ancestral strain. ' $R$ ' identifies a virus-resistant strain; the number following $R$ identifies the virus for which a bacterial strain evolved resistance. White boxes indicate sensitive phenotypes; gray boxes indicate resistant phenotypes. The sensitivityresistance matrix was determined on two occasions; once at the beginning of the experiment $\left(t_{0}\right)$ and 12 months later $\left(t_{f}\right) . \Delta$ Indicates that a bacterial strain's phenotype changed between the time that the two sensitivity-resistance matrices were constructed $\left(^{+}=\right.$strain died during 12 month period; second profile not constructed). The bottom two rows represent total resistance, which was calculated at $t_{0}$ and $t_{f}$, by summing the number of resistant phenotypes (gray boxes) in each column.

reduced when cultured under low phosphorus concentrations $(P=0.014$, nutrient main effect, two-way ANOVA, Figure 2). There was, however, no resistance $\times$ nutrient interaction $(P=0.342$, twoway ANOVA, Figure 2). Therefore, Synechococcus experienced a COR resulting in an average $20 \%$ reduction in relative fitness, irrespective of phosphorus availability.

The second competition experiment revealed there were significant differences among sensitive and virus-resistant strains of WH8018. Oneway ANOVA followed by post hoc comparison of means indicated that growth rates among the WH8018 populations were marginally different $\left(P_{\text {null }}=0.0717\right)$. When linear contrasts were performed, resistant strains of Synechococcus had significantly lower growth rates than the ancestral population $\left(P_{\text {contrast }}=0.0499\right.$, Figure 3$)$, equating to an $18 \%$ reduction in relative fitness.
Similar to the results from the growth curve data, competition experiments revealed that Synechococcus growth rates were not affected by total resistance $(P=0.58$, Figure 4, Table 1). In contrast, Synechococcus growth rates were affected by compositional resistance $(P=0.023$, Figure 4 , Table 1$)$. Specifically, eigenvectors corresponding with PCoA axis 1, which explained $39.5 \%$ of the compositional variation in BOR for WH8018 strains, were strong predictors of Synechococcus growth rate $\left(r^{2}=0.54\right.$, $P=0.023$, Table 1, Figure 4). This pattern appears to have been driven in part by the sensitivity and resistance of bacteria to a few viral isolates. For example, S-RIM4 and S-RIM11 had strong negative correlations with PCoA axis $1(r=-0.94$ and -0.80 , respectively), while S-RIM7 and S-RIM20 both had a strong positive correlation with PCoA axis 1 $(r=0.91)$. In other words, the relationship between compositional resistance and Synechococcus 

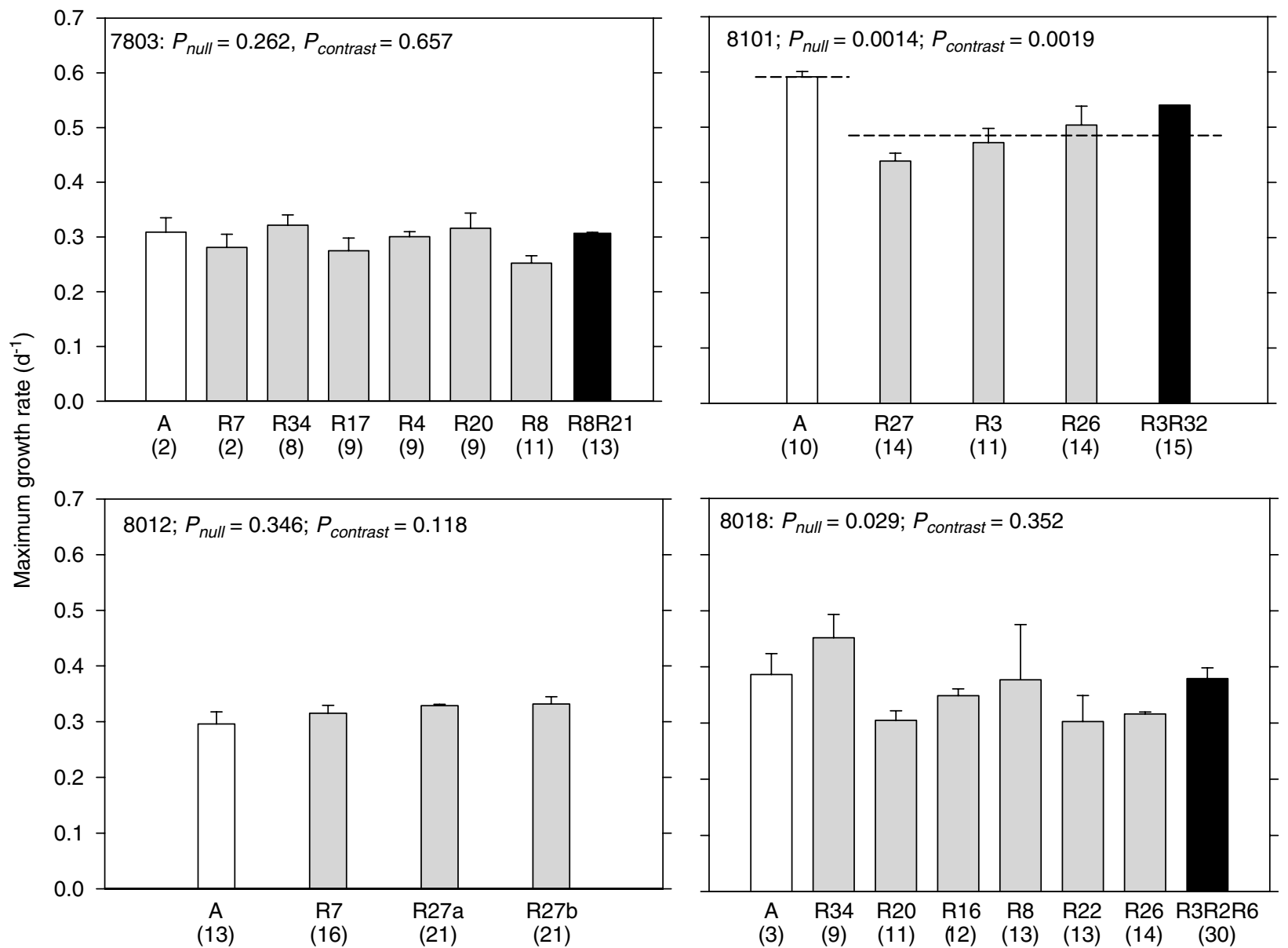

Figure 1 Maximum growth rates $\left(\mu_{\max }\right)$ for ancestral (white bars) and virus-resistant strains (gray and black bars) of marine Synechococcus. Strains that went through one viral selection are colored gray; strains that went through multiple $(\geqslant 2)$ viral selections are colored black. The four panels display results for the different phylogenetic groups of Synechococcus (WH7803, WH8101, WH8012 and WH8018). $P_{\text {null }}$ refers to statistical probability that $\mu_{\max }$ of all strains within a phylogenetic group are equal. $P_{\text {contrast }}$ refers to the statistical probability that the $\mu_{\max }$ values of virus-resistant strains are less than that of the sensitive strain. Vertical bar data represent mean + s.e.m. Dashed horizontal lines in the WH8101 panel represent average $\mu_{\max }$ values generated from the ANOVA model with linear contrasts (that is, ancestral vs resistant). Letters below the vertical bars identify whether a strain is ancestral (A) or if is resistant (R); if resistant, the letter is followed by the number of the viral isolate for which a bacterial strain evolved resistance. Numbers in parentheses indicates the total resistance for a bacterial strain (that is, number of viruses in a BOR profile to which a strain is resistant). R27a and R27b represent resistant Synechococcus strains isolated after exposure to same virus (S-RIM27).

growth rate was driven by the relatively low fitness of strains that were sensitive to S-RIM4 and S-RIM11 and relatively high fitness of strains that were resistant to S-RIM7 and S-RIM20 (Table 2, Figure 4).

\section{COR: assessing detection limits}

As expected, the power to detect a COR increased with sample size and the magnitude of the COR (expressed as the percent reduction in relative fitness). Compared to the competition assays, power increased more rapidly with COR in the growth curve experiments (Figures 5a and b). This reflects an underlying difference in measurement error. For example, the average coefficient of variation $(\mathrm{CV}=$ standard deviation/mean) in the competition assays was 0.21 , while the average CV in the growth curve experiments was only 0.10. Increasing the number of replicates from 3 to 5 would have increased our ability to detect a $10 \%$ reduction in relative fitness by a factor of two, but we had sufficiently high power (0.79) to detect a $20 \%$ reduction in relative fitness with our experimental design (Figure 5a). In competition assays, increasing the number of replicates from 5 to 10 would have increased our power to detect a $10 \%$ reduction in relative fitness from 0.17 to 0.36 (Figure 5b). Irrespective of the sample size $(n=3-10)$, however, power was relatively low $(\leqslant 0.13)$ when we simulated small reductions in relative fitness $(\leqslant 5 \%$, Figure $5 \mathrm{~b})$.

\section{Discussion}

It is generally assumed that virus-resistant bacteria have a competitive advantage over sensitive bacteria in the presence of an infectious virus (Bohannan 
Table 2 Relationships between BOR and COR for different phylogenetic groups of marine Synechococcus

\begin{tabular}{|c|c|c|c|c|c|}
\hline Group & COR experiment & $\mathrm{n}$ & $r^{2}$ & $\mathrm{P}$-value & \\
\hline WH7803 & Growth curves & 8 & 0.008 & 0.829 & \\
\hline WH8012 & Growth curves & 4 & 0.299 & 0.453 & \\
\hline WH8101 & Growth curves & 5 & 0.131 & 0.549 & \\
\hline \multirow{2}{*}{ WH8018 } & Growth curves & 9 & 0.006 & 0.857 & \\
\hline & Competition & 9 & 0.044 & 0.620 & \\
\hline Group & Response & PCoA Axis 1 & PCoA Axis 2 & PCoA Axis 3 & Cumulative \\
\hline \multirow[t]{3}{*}{ WH7803 } & \% Variance explained & 44.8 & 22.2 & 15.4 & 82.5 \\
\hline & $P$-value (growth curves) & 0.837 & 0.230 & 0.760 & \\
\hline & $r^{2}$ & 0.008 & 0.230 & 0.017 & \\
\hline \multirow[t]{3}{*}{ WH8012 } & \% Variance explained & 42.4 & 38.1 & 19.5 & 100.0 \\
\hline & $P$-value (growth curves) & 0.599 & 0.735 & 0.123 & \\
\hline & $r^{2}$ & 0.161 & 0.407 & 0.248 & \\
\hline \multirow[t]{3}{*}{ WH8101 } & \% Variance explained & 39.0 & 35.3 & 15.2 & 89.5 \\
\hline & $P$-value (growth curves) & 0.873 & 0.247 & 0.393 & \\
\hline & $r^{2}-0-3 x$ & 0.010 & 0.407 & 0.248 & \\
\hline \multirow[t]{5}{*}{ WH8018 } & \% Variance explained & 39.5 & 30.0 & 15.1 & 84.6 \\
\hline & $P$-value (growth curves) & 0.146 & 0.2311 & 0.834 & \\
\hline & $r^{2}$ & 0.318 & 0.228 & 0.008 & \\
\hline & $P$-value (competitions) & 0.023 & 0.426 & 0.694 & \\
\hline & $r^{2}$ & 0.548 & 0.092 & 0.023 & \\
\hline
\end{tabular}

Abbreviations: COR, cost of resistance; BOR, breadth of resistance; PCoA, Principal Coordinates Analysis.

Top: results from linear regression testing for an effect of total resistance on bacterial growth rate. COR experiment indicates whether growth rate data was generated from growth curves $\left(\mu_{\max }\right)$ or competition experiments. Bottom: results from PCoA and linear regression testing for an effect of compositional resistance on bacterial growth rate. The first three axes from a PCoA were used to explain variation ('Cumulative'=sum of first three axes) in the sensitivity-resistance matrices of the different Synechococcus groups. In addition, eigenvectors from the first three axes of a PCoA analysis were used as predictors of Synechococcus growth rate in linear regression analyses. $n=$ sample size, $r^{2}=$ coefficient of determination from regression analysis, $P$-value = probability that slope from regression analysis is different from zero.

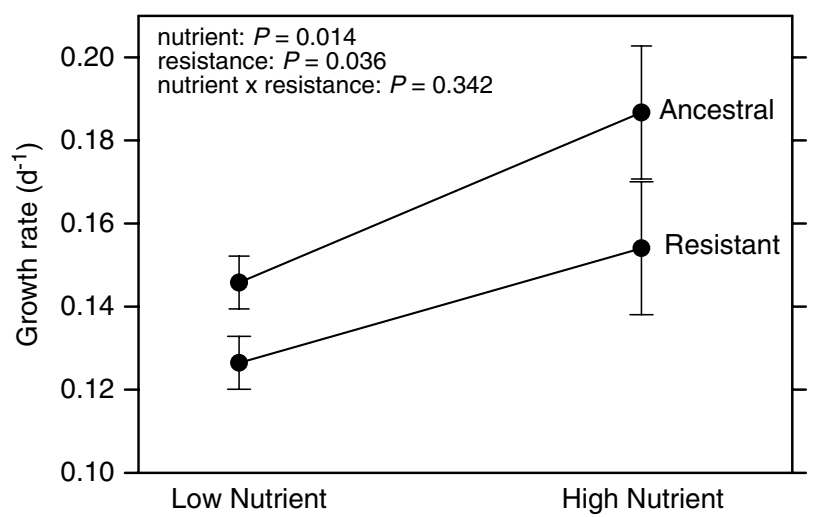

Figure 2 Growth rates for ancestral (WH7803) and virusresistant (WH7803R8) strains of Synechococcus from a competition experiment designed to test whether COR was dependent on resource concentrations. Low nutrient $=0.9 \mu \mathrm{M} \quad \mathrm{PO}_{4}$; high nutrient $=90 \mu \mathrm{M} \mathrm{PO}_{4}$. $P$-values were generated from a two-way ANOVA model.

and Lenski, 2000). Similarly, it is assumed that resistant and sensitive bacteria are able to coexist because the evolution of virus resistance comes at a fitness cost (Waterbury and Valois, 1993; Bohannan and Lenski, 2000). This trade-off may maintain genetic and phenotypic diversity in bacterial populations and thus dampen the impacts of viruses on microbial food webs (Middelboe et al., 2001). Our study provides the first evidence for a cost of virus

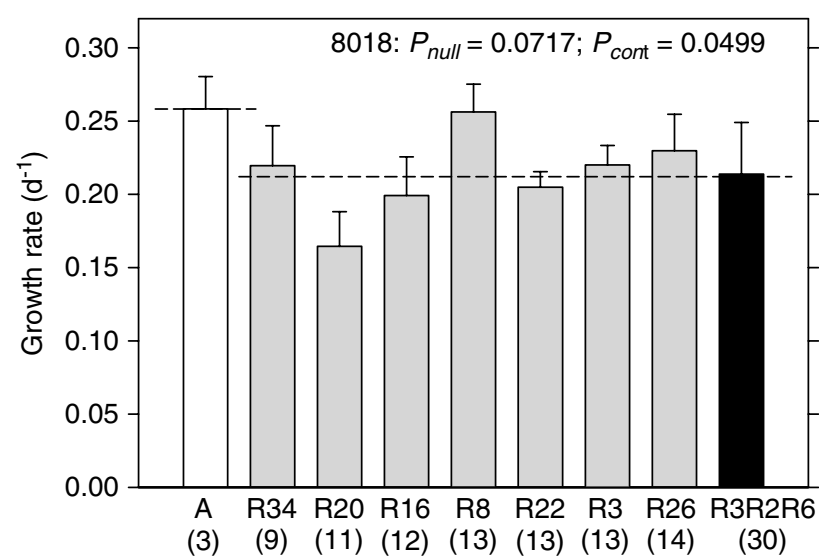

Figure 3 Growth rates from a competition experiment to test for fitness differences among ancestral (white bars) and virusresistant strains (gray and black bars) of Synechococcus WH8018. Vertical bar data represent mean \pm s.e.m. The dashed horizontal line represents the average growth rate generated from the ANOVA model with linear contrasts (that is, ancestral vs resistant). Letters below the vertical bars identify whether a strain is ancestral (A) or if is resistant (R); if resistant, the letter is followed by the number of the viral isolate for which a bacterial strain evolved resistance. Numbers in parentheses indicates the total resistance for a bacterial strain (that is, number of viruses in a BOR profile to which a strain is resistant).

resistance in marine bacteria. A COR was only found in a few of our experiments. When detected, however, it resulted in an $18-20 \%$ reduction in relative fitness. This cost of virus resistance is 

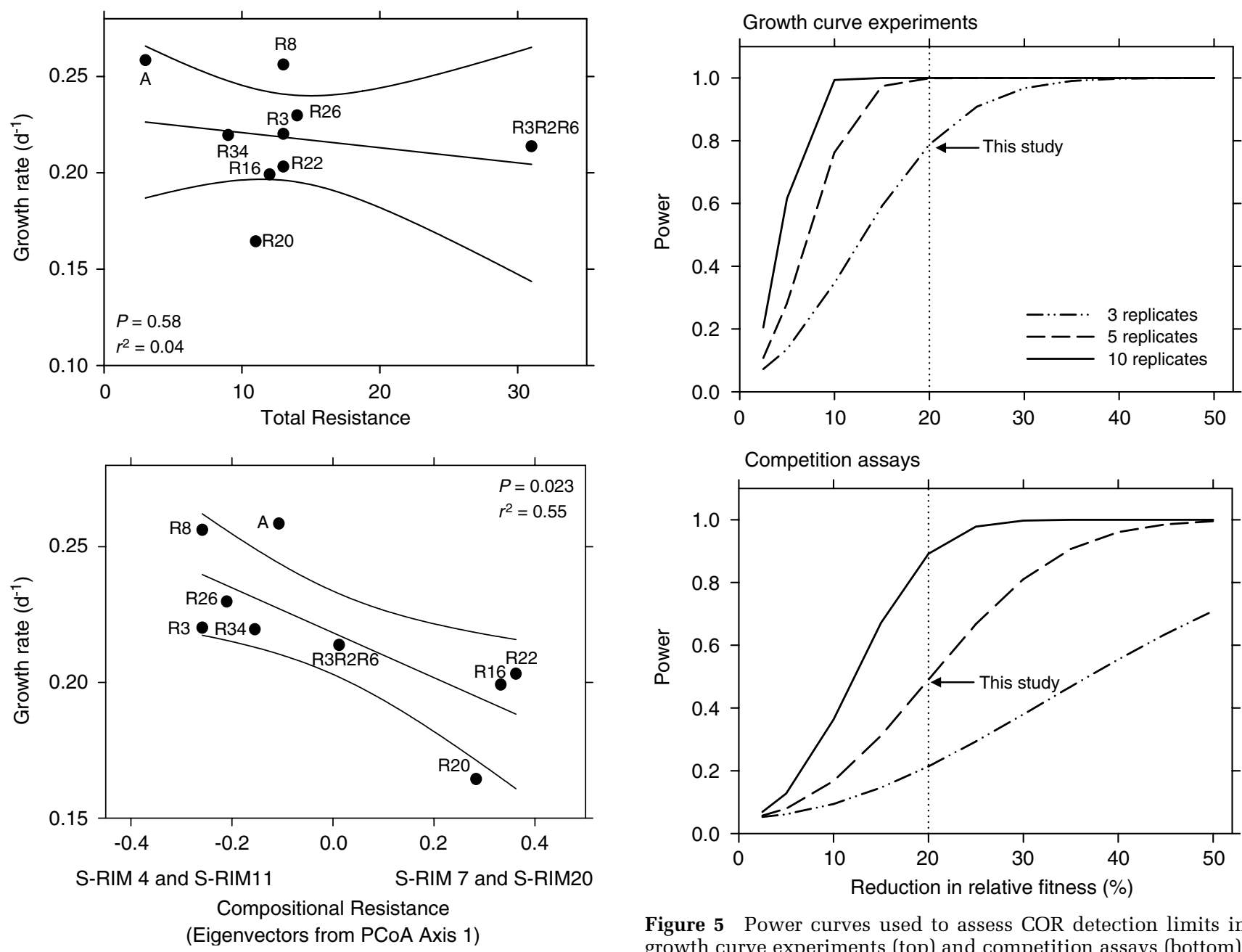

Figure 4 Growth rates from a competition experiment to test for an effect of breadth-of-resistance (BOR) on Synechococcus fitness. Top panel: total resistance (number of viruses to which a Synechococcus strain was resistant) in a BOR profile was a poor predictor of mean $(n=5)$ Synechococcus growth rate. Bottom panel: compositional resistance of a strain's BOR was a significant predictor of mean $(n=5)$ Synechococcus growth rate. Compositional resistance was calculated by generating a similarity matrix from a binary sensitivity-resistance matrix (Table 1). We then performed a Principal Coordinates Analysis (PCoA) on the similarity matrix and used the resulting eigenvectors from the first PCoA axis as predictors of Synechococcus growth rate. In this case, the eigenvectors from the first PcoA axis were used (see Table 1). Letters next to data points indicate whether a strain is ancestral (A) or if is resistant (R); if resistant, the letter is followed by the number of the viral isolate for which a bacterial strain evolved resistance.

in-line with the fitness reductions reported in other bacteria-phage systems (Lenski, 1988a). Moreover, we observed that a bacterial strain's growth rate was affected by the identity of the different viruses to which it evolved resistance, as described by its BOR profile. These results are important because the magnitude of a strain's COR has the potential to modify competitive outcomes and influence microbial community composition (Bohannan et al., 2002).

Figure 5 Power curves used to assess COR detection limits in growth curve experiments (top) and competition assays (bottom). Power is plotted as a function of the reduction in relative fitness (that is, COR) for three different levels of replication $(n=3,5,10)$. The dashed vertical line at $20 \%$ reduction in relative fitness represents the COR magnitude detected in some of our experiments.

Detecting a COR in Synechococcus

A COR was only detected in half of our experiments. An extensive review of the plant literature revealed that a cost of pathogen or herbivore resistance was only found half of the investigated cases (Bergelson and Purrington, 1996). Likewise, a fitness cost is not always apparent in bacteria that have evolved resistance to antibiotics (Lenski, 1998; Gagneux et al., 2006) or viruses (Lythgoe and Chao, 2003; Brockhurst et al., 2005). As theory predicts, the absence of a COR can result in the extinction of both the ancestral bacterial host and virus as in the case of Escherichia coli and T5 phage (Lenski and Levin, 1985).

There are a number of potential explanations for the lack of a COR in some of our experiments. First, a COR does not have to be large to be biologically important (Sasaki, 2000; Bohannan et al., 2002). There are, however, constraints on our ability to detect a small COR. For example, our power 
analyses suggest that it is difficult to detect small fitness costs, even when measurement error is relatively low (Figures 5a and b). Increased statistical power can be achieved through increased replication, but this strategy will often be met by a reduction in the number of experimental treatments or strains that can be investigated (Cottingham et al., 2005). Thus, our results suggest that the difficulty of detecting a COR - in this study and in other systems - may be due in part to the statistical challenge of detecting small, but meaningful biological effect sizes.

Second, the cost of virus resistance can be affected by genotype $\times$ environment interactions (Bohannan and Lenski, 2000), such that relative fitness is reduced under particular environmental conditions. In plant systems, a COR to herbivory often involves increased allocation of nutrients to secondary compounds used to deter grazing pressure (Bergelson and Purrington, 1996; Siemens et al., 2003), suggesting that a COR may be expressed more in nutrient-limited environments. For bacteria, it is assumed that virus resistance often involves the modification of pilus structure or another surface receptor molecule (Lythgoe and Chao, 2003; Mizoguchi et al., 2003). Such mutations may decrease host fitness by reducing nutrient uptake rates, thus influencing competitive interactions. In E. coli, for example, mutations that confer resistance to virulent lambda phage usually modify the LamB protein (Szmelcman and Hofnung, 1975), which is involved in the uptake of maltose and trehalose, but not glucose (Travisano and Lenski, 1996). Thus, the COR to virulent lambda phage is dependent upon whether it is supplied with glucose or trehalose (Bohannan et al., 1999). We tested the hypothesis that the COR in Synechococcus would be greater under reduced phosphorus concentrations, since phosphorus limitation has been implicated in viral infection dynamics (Wilson et al., 1996). Synechococcus growth rates were reduced under low phosphorus concentrations, but this had no effect on the magnitude of the COR for the resistant strain we tested. This suggests that some viruses may target Synechococcus receptors that are not involved in phosphorus acquisition. Future studies should address whether other environmental variables, such as nitrogen concentrations, temperature or light levels influence the COR to viruses in marine bacteria.

There is also evidence that a COR may be expressed more under competitive conditions. For example, the COR for T4-resistant E. coli was half the magnitude in batch culture than continuous culture, where it is assumed that bacteria experience more intense resource competition (Bohannan et al., 2002). Results from our experiments with Synechococcus are consistent with this view; we were unable to detect a COR in growth curve experiments with WH8018 strains, but measured an 18\% reduction in relative fitness with the same strains in competition assays.
Third, the COR can be eliminated if resistant strains are capable of reverting to a sensitive phenotype. Initially, no COR was detected in studies of virus-resistant Pseudomonas strains (Brockhurst et al., 2005). Subsequent experiments within the same study revealed that certain resistant strains were reverting to a sensitive phenotype in $<24 \mathrm{~h}$, thus eliminating the COR. This reversion pattern, however, was not observed for other strains of Pseudomonas that had evolved resistance to different viruses (Brockhurst et al., 2005). In this study, Synechococcus BOR profiles were generally stable over time under our culturing conditions, suggesting that reversions did not play a large role in altering a strain's COR over the course of our experiment. Of the 134 resistant phenotypes tested, only 6\% reverted to a sensitive phenotype. Of those reversions, most were associated with two viruses, suggesting that some resistance mutations are less stable than others. Nevertheless, reversions may not be rare; Synechococcus infections were more common when host strains had been cultured in the absence of viruses for at least 2 years, during which time they presumably lost their resistance (Waterbury and Valois, 1993).

In addition to reversions, the COR can be diminished through time owing to compensatory mutations. Compensatory mutations refer to host mutations at secondary loci that increase fitness independent of the mutation involved in viral resistance (Wijngaarden et al., 2005). For example, the COR for T4-resistant E. coli declined by $\sim 50 \%$ over 400 generations in the absence of T4 (Lenski, 1988b). Although potentially important, our study was not designed to test for the effect of compensatory mutations on the COR in marine Synechococcus.

Lastly, there is some theoretical evidence to suggest that a COR is not required for the coexistence of virus-sensitive and virus-resistant bacterial populations. For example, metapopulation models, which include spatial heterogeneity allow for the coexistence of host and parasite populations without assuming a COR (Thrall and Burdon, 2000). Similarly, gene-for-gene models with finite population sizes can maintain phenotypic diversity through genetic drift and periods of selective neutrality (Salathe et al., 2005). To our knowledge, these models have not yet been tested. Also, it is possible that some viral 'infections' actually increase host fitness. For example, it has been hypothesized that viruses may be a source of nutrition and novel genetic information for marine bacteria (Fuhrman, 1999).

\section{COR affected by compositional resistance}

Previous studies suggest that the number of viruses to which a bacterium has evolved resistance will influence the COR. For example, the COR in E. coli is greater when it has evolved resistance to both T4 
and T7, instead of T4 alone (Lenski, 1988a). Therefore, we predicted that the COR would increase with total resistance, which we defined as the number of viruses that a Synechococcus strain was resistant to out of the 32 viral isolates tested in this study. Despite substantial variation in the predictor and response variables, total resistance was not positively correlated with a strain's fitness. Pleiotropy, the effect of one mutation on multiple phenotypes, is one explanation for the lack of a relationship between total resistance and fitness (Lenski, 1988a). For example, E. coli resistance to colicins arises nonindependently, such that bacterial resistance to one colicin type confers resistance to additional colicin types (Feldgarden and Riley, 1999). Similarly, on average, marine Synechococcus gained resistance to eight additional viruses when challenged against a single viral isolate (Stoddard et al., unpublished data). Therefore, total resistance may not reflect the number of viruses to which a host has become independently resistant. This non-additive effect was especially apparent with the observation that Synechococcus fitness did not decline after going through multiple, independent selections for virus resistance (black bars in Figures 1 and 3).

Under competitive conditions, however, Synechococcus growth rate could be explained in part by the compositional resistance observed in BOR profiles. In other words, although fitness was unaffected by total resistance, it was affected by the identity of the specific viruses to which it evolved resistance. In particular, it appears that WH8018 resistance to S-RIM7 and S-RIM20 comes at a particularly large cost. As in E. coli, it is possible that Synechococcus resistance to these viral isolates involves mutations that result in a modified physiology that is particularly costly (Lenski, 1988a). Future studies should evaluate whether different mechanisms of virus resistance (for example, modifications of different receptor molecules) lead to different fitness costs in marine Synechococcus. Together, our results have important implications for understanding the diversity and function of marine microbial food webs. In theory, even if small, a COR can allow for the coexistence sensitive bacteria, resistant bacteria and a virus population (Bohannan and Lenski, 2000). Hence, by detecting a significant COR in some of our experiments, our results are consistent with the view that viruses play a role in promoting Synechococcus diversity in marine waters (Muhling et al., 2005). Results from our study also provide insight into potential evolutionary controls on the virusinduced mortality of marine bacteria. Specifically, our results suggest that the composition of cooccurring viruses may influence virus-induced mortality rates via varied fitness costs. For example, if on average, the viruses present in a microbial community impose a small COR, then resistant bacteria should be common and lead to lower viral abundances and lower virus-induced mortality. In contrast, if the viral community imposes a high
COR, then resistant strains of bacteria should be relatively rare and lead to higher viral abundances and higher virus-induced mortality. Thus, variation in fitness costs associated with virus resistance has the potential to structure microbial communities and regulate biogeochemical cycles in marine ecosystems.

\section{Acknowledgements}

We thank C Jessup and AE Wilson for critical review of the paper. We acknowledge R Creton, J Nathanson, J Wong and the Leduc Bioimaging Facility at Brown University for assistance with confocal microscopy used for developing our filters sets for the competition experiments; M Tatar for plate reader access; J Casey preliminary lab work; A Michener for preliminary data analysis; K Mulder for statistical advice. This study was supported by the National Science Foundation (OCE-0315645 and OCE0314523) and the Gordon and Betty Moore Foundation. This paper is dedicated to the memory of Alison Michener, an aspiring young scientist with a knack for enriching Synechococcus. Kellogg Biological Station contribution number 1420.

\section{References}

Baty F, Delignette-Muller ML. (2004). Estimating the bacterial lag time: which model, which precision? Int J Food Microbiol 91: 261-277.

Bergelson J, Purrington CB. (1996). Surveying patterns in the cost of resistance in plants. Am Nat 148: 536-558.

Bertilsson S, Berglund O, Karl DM, Chisholm SW. (2003). Elemental composition of marine Prochlorococcus and Synechococcus: implications for the ecological stoichiometry of the sea. Limnol Oceanogr 48: 17211731.

Bohannan BJM, Kerr B, Jessup CM, Hughes JB, Sandvik G. (2002). Trade-offs and coexistence in microbial microcosms. Anton Leeuw Int J G 81: 107-115.

Bohannan BJM, Lenski RE. (2000). Linking genetic change to community evolution: insights from studies of bacteria and bacteriophage. Ecol Lett 3: 362-377.

Bohannan BJM, Travisano M, Lenski RE. (1999). Epistatic interactions can lower the cost of resistance to multiple consumers. Evolution 53: 292-295.

Bouvier T, del Giorgio PA. (2007). Key role of selective viral-induced mortality in determining marine bacterial community composition. Environ Microbiol 9: 287-297.

Brahamsha B. (1996). A genetic manipulation system for oceanic cyanobacteria of the genus Synechococcus. Appl Environ Microbiol 62: 1747-1751.

Brockhurst MA, Buckling A, Rainey PB. (2005). The effect of a bacteriophage on diversification of the opportunistic bacterial pathogen, Pseudomonas aeruginosa. Proc R Soc Lond, B 272: 1385-1391.

Buckling A, Rainey PB. (2002). Antagonistic coevolution between a bacterium and a bacteriophage. Proc $R$ Soc Lond, B 269: 931-936.

Chao L, Levin BR, Stewar FM. (1977). A complex community in a simple habitat: an experimental study with bacteria and phage. Ecology 58: 369-378.

Cottingham KL, Lennon JT, Brown BL. (2005). Knowing when to draw the line: designing more informative 
ecological experiments. Front Ecol Environ 3: 145-152.

de Roode JC, Pansini R, Cheesman SJ, Helinski MEH, Huijben S, Wargo AR et al. (2005). Virulence and competitive ability in genetically diverse malaria infections. Proc Natl Acad Sci USA 102: 7624-7628.

Feldgarden M, Riley MA. (1999). The phenotypic and fitness effects of colicln resistance in Escherichia coli K-12. Evolution 53: 1019-1027.

Fuhrman JA. (1999). Marine viruses and their biogeochemical and ecological effects. Nature 399: $541-548$

Fuhrman JA, Noble RT. (1995). Viruses and protists cause similar bacterial mortality in coastal seawater. Limnol Oceanogr 40: 1236-1242.

Gagneux S, Long CD, Small PM, Van T, Schoolnik GK, Bohannan BJM. (2006). The competitive cost of antibiotic resistance in Mycobacterium tuberculosis. Science 312: 1944-1946.

Gotelli NJ, Ellison AM. (2004). A Primer of Ecological Statistics. Sinauer: Sunderland, pp 510.

Harcombe WR, Bull JJ. (2005). Impact of phages on twospecies bacterial communities. Appl Environ Microbiol 71: 5254-5259.

Inoue T, Matsuzaki S, Tanaka S. (1995). A 26-kDa outermembrane protein, OmpK, common to Vibrio species is the receptor for a broad-host-range vibriophage, KVP40. FEMS Microbiol Lett 125: 101-105.

Jiang S, Fu W, Chu W, Fuhrman JA. (2003). The vertical distribution and diversity of marine bacteriophage at a station off Southern California. Microb Ecol 45 : 399-410.

Kassen R, Rainey PB. (2004). The ecology and genetics of microbial diversity. Annu Rev Microbiol 58: 207-231.

Legendre P, Legendre L. (1998). Numerical Ecology. Elsevier: Amsterdam, pp 853.

Lenski RE. (1988a). Experimental studies of pleiotropy and epistasis in Escherichia coli 1. variation in competitive fitness among mutants resistant to virusT4. Evolution 42: 425-432.

Lenski RE. (1988b). Experimental studies of pleiotropy and epistasis in Escherichia coli 2. compensation for maladaptive effects associated with resistance to virus-T4. Evolution 42: 433-440.

Lenski RE. (1998). Bacterial evolution and the cost of antibiotic resistance. Int Microbiol 1: 265-270.

Lenski RE, Levin BR. (1985). Constraints on the coevolution of bacteria and virulent phage - a model, some experiments, and predictions for natural communities. Am Nat 125: 585-602.

Lythgoe KA, Chao L. (2003). Mechanisms of coexistence of a bacteria and a bacteriophage in a spatially homogeneous environment. Ecol Lett 6: 326-334.

Mann NH. (2003). Phages of the marine cyanobacterial picophytoplankton. FEM Microbiol Rev 27: 17-34.

Marston MF, Sallee JL. (2003). Genetic diversity and temporal variation in the cyanophage community infecting marine Synechococcus species in Rhode Island's coastal waters. Appl Environ Microbiol 69: 4639-4647.

McDaniel L, Paul JH. (2005). Effect of nutrient addition and environmental factors on prophage induction in natural populations of marine Synechococcus species. Appl Environ Microbiol 71: 842-850.

Middelboe M, Hagstrom A, Blackburn N, Sinn B, Fischer U, Borch NH et al. (2001). Effects of bacterio- phages on the population dynamics of four strains of pelagic marine bacteria. Microb Ecol 42: 395-406.

Mizoguchi K, Morita M, Fischer CR, Yoichi M, Tanji Y, Unno H. (2003). Coevolution of bacteriophage PP01 and Escherichia coli O157 : H7 in continuous culture. Appl Environ Microbiol 69: 170-176.

Muhling M, Fuller NJ, Millard A, Somerfield PJ, Marie D, Wilson WH et al. (2005). Genetic diversity of marine Synechococcus and co-occurring cyanophage communities: evidence for viral control of phytoplankton. Environ Microbiol 7: 499-508.

Paul JH, Sullivan MB. (2005). Marine phage genomics: what have we learned? Curr Opin Biotechnol 16: 299-307.

Proctor LM, Fuhrman JA. (1990). Viral mortality of marine bacteria and cyanobacteria. Nature 343: 60-62.

Rocap G, Distel DL, Waterbury JB, Chisholm SW. (2002). Resolution of Prochlorococcus and Synechococcus ecotypes by using 16S-23S ribosomal DNA internal transcribed spacer sequences. Appl Environ Microbiol 68: 1180-1191.

Salathe M, Scherer A, Bonhoeffer S. (2005). Neutral drift and polymorphism in gene-for-gene systems. Ecol Lett 8: 925-932.

Sasaki A. (2000). Host-parasite coevolution in a multilocus gene-for-gene system. Proc $R$ Soc Lond, B 267: 2183-2188.

Siemens DH, Lischke H, Maggiulli N, Schurch S, Roy BA. (2003). Cost of resistance and tolerance under competition: the defense-stress benefit hypothesis. Evol Ecol 17: $247-263$.

Stomp M, Huisman J, de Jongh F, Veraart AJ, Gerla D, Rijkeboer $\mathrm{M}$ et al. (2004). Adaptive divergence in pigment composition promotes phytoplankton biodiversity. Nature 432: 104-107.

Sullivan MB, Waterbury JB, Chisholm SW. (2003). Cyanophages infecting the oceanic cyanobacterium Prochlorococcus. Nature 424: 1047-1051.

Suttle CA, Chan AM. (1994). Dynamics and distribution of cyanophages and their effect on marine Synechococcus spp. Appl Environ Microbiol 60: 3167-3174.

Szmelcman S, Hofnung M. (1975). Maltose transport in Escherichia coli K-12-involvement of bacteriophagelambda receptor. J Bact 124: 112-118.

Thrall PH, Burdon JJ. (2000). Effect of resistance variation in a natural plant host-pathogen metapopulation on disease dynamics. Plant Pathol 49: 767-773.

Travisano M, Lenski RE. (1996). Long-term experimental evolution in Escherichia coli.4. Targets of selection and the specificity of adaptation. Genetics 143: 15-26.

Waterbury JB, Valois FW. (1993). Resistance to cooccurring phages enables marine Synechococcus communities to coexist with cyanophages abundant in seawater. Appl Environ Microbiol 59: 3393-3399.

Waterbury JB, Valois FW, Franks DG. (1986). Biological and ecological characterization of the marine unicellular cyanobacterium Synechococcus. Can Bull Fish Aquat Sci 214: 71-120.

Waterbury JB, Willey JM. (1988). Isolation and growth of marine planktonic cyanobacteria. Methods Enzymol 167: 100-105.

Weinbauer MG. (2004). Ecology of prokaryotic viruses. FEMS Microbiol Rev 28: 127-181. 
Weinbauer MG, Rassoulzadegan F. (2004). Are viruses driving microbial diversification and diversity? Environ Microbiol 6: 1-11.

Wijngaarden PJ, van den Bosch F, Jeger MJ, Hoekstra RF. (2005). Adaptation to the cost of resistance: a model of compensation, recombination, and selection in a haploid organism. Proc $R$ Soc Lond, B 272: 85-89.

Wilhelm SW, Suttle CA. (1999). Viruses and nutrient cycles in the sea - viruses play critical roles in the

structure and function of aquatic food webs. Bioscience 49: 781-788.

Wilson WH, Carr NG, Mann NH. (1996). The effect of phosphate status on the kinetics of cyanophage infection in the oceanic cyanobacterium Synechococcus sp WH7803. J Phycol 32: 506-516.

Zwietering MH, Jongenburger I, Rombouts FM, Vantriet $\mathrm{K}$. (1990). Modeling of the bacterial-growth curve. Appl Environ Microbiol 56: 1875-1881. 